Bulletin

of the

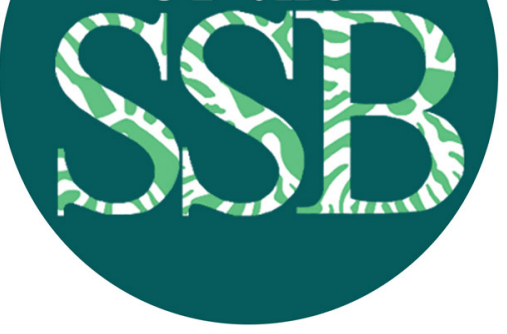

\title{
A new framework for assessing the contributions of professionals in the natural sciences
}

Lauren A. Esposito', Marymegan Daly², Matthew K. Fujita ${ }^{3}$, Jacob A. Gorneau ${ }^{1,4}$, Giovanni Rapacciuolo', Luiz Rocha', Lauren Scheinberg', Jessica Ware ${ }^{5}$, Corey K. Welch ${ }^{6}$, Alison N. Young ${ }^{1}$, Rayna C. Bell ${ }^{1}$

Published: 27 January 2022

\section{Affiliations:}

${ }^{1}$ Institute for Biodiversity Science and Sustainability, California Academy of Sciences, San Francisco, CA 94118 USA; ${ }^{2}$ Department of Evolution, Ecology \& Organismal Biology, The Ohio State University, Columbus, Ohio, USA; ${ }^{3}$ Amphibian and Reptile Diversity Research Center, Department of Biology, The University of Texas at Arlington, Arlington, TX 76019, USA; ${ }^{4}$ Department of Biology, San Francisco State University, San Francisco, CA 94132, USA; ${ }^{5}$ Invertebrate Zoology, American Museum of Natural History, New York, NY 10026, USA; ${ }^{6}$ STEM Scholars Program, Iowa State University, Ames, IA 50010, USA

Correspondence:

Lauren Esposito

Email: lesposito@calacademy. org
Keywords: scientific success; diversity; equity; inclusion; mentorship

\section{INTRODUCTION}

The foundations of the natural sciences are intertwined with the histories of racism and colonialism, including the explicit distortion of scientific discoveries in the formulation and justification of racist theories of humanity as well as the biases and harmful actions that persist in our present-day workplaces (Haraway 1984; Graves Jr. 2004; Graves Jr. 2015; Das and Lowe 2018; Graves Jr. 2019; Davies et al. 2021; Cronin et al. 2021). Many individuals, institutions, and professional societies in the natural sciences are committed to implementing anti-racist actions and policies as the foundation of a future that is equitable and that truly reflects our values. Yet, the work of diversity, equity, and inclusion is undervalued and disproportionately falls to marginalized groups, further hindering their academic "success" under current metric systems. This inequity compounds the burden of the legacies of racism with the urgent need to dismantle them.

Under current hiring, retention, promotion, and tenure practices (HRPT), "service" (including diversity, equity, and inclusion (DEI) research) is often considered a low value activity, and rather than being viewed as a positive professional contribution can instead be discriminatory to 
minoritized and historically excluded faculty who typically engage in more service than their peers (Olsen et al. 1995; Harley 2008; Alperin et al. 2019; Jimenez et al. 2019). In addition, many activities (i.e., social media science communication) that could be viewed under the umbrella of "service" were unheard of 10-15 years ago (i.e., 2-3 tenure cycles), and may have no mechanism for formal recognition or be dismissed entirely as non-professional or extra-curricular. Lieff (2009), in focusing on similar challenges in academic medicine, suggested a professional development activity including written reflection on (personal and professional) values and goals. They argued this will lead to more meaningful and well-aligned activities for academics to be evaluated by, which in turn will improve recruitment and retention.

Acknowledging and rewarding professional activities that we value, that reduce barriers to participation in our disciplines, and which promote a sense of belonging for scientists of all backgrounds is a first step towards redefining the traditional concept and ascribed value of "service". It is also worth noting that ecology and evolutionary biology are particularly egregious in lack of diversity relative to other STEM fields (Cronin et al. 2021), and so the need for this work remains critical. We propose that our disciplines adopt and promote an inclusive model of science with an expanded view of impact that contributes broadly to a more equitable and accessible science (as suggested by Davies et al. 2021; see also discussions on drawbacks/limitations of existing academic ranking/metric systems in Gruber 2014; Fire and Guestrin 2019). Our proposed framework for assessment incorporates diverse perspectives, includes meaningful and equitable community partnerships, and promotes impactful mentorship. It differs from current models in differentiating the foci and constituencies for service activities and in explicitly connecting all professional activities under a framework of advancing scientific knowledge. Embedded within each element of this framework is a call to reflect upon what individuals have personally done to make science more diverse, equitable, and inclusive-moving the work of DEI away from the shadows and off the shoulders of the few and ascribing direct value to traditional service work along with a culture of personal responsibility in the promotion of DEI.

As ecologists and evolutionary biologists, the authors of this proposed framework are also acutely aware that we are in the midst of a global biodiversity crisis, and yet our current HRPT practices cannot incentivize or recognize many of the actions needed within the scientific community to avert this crisis. Although research in our fields has the potential to inform how best to protect biodiversity, the current metrics by which we assess scientific success as individuals, institutions, and as a broader scientific community neither reflect nor incentivize behaviors that would avert or mitigate the biodiversity crisis (see Gorneau et al. 2022 for further discussion in the context of monography). Further, we recognize that we are in a societal crisis of justice and equity with impacts that reverberate within our discipline and that are amplified by differences in the way identity influences how professional activities are viewed (Corneille et al. 2019; Jimenez et al. 2019; Evangelista et al. 2020; Miner et al. 2019; Miriti 2020; Orfinger 2020; Maas et al. 2021). How can our scientific communities shift to measuring and rewarding activities that we value rather than valuing and rewarding only what we can easily measure? This flexible proposed framework addresses these challenges with more explicit and granular consideration of 
professional activities and allows for changes in focus across career stage and career trajectory, providing a more inclusive view of the impacts one can have over the course of their career. We believe that this approach will be more effective for acknowledging and rewarding efforts in line with our scientific and institutional values, rather than a narrow focus on only the traditional performance metrics (publications, grants, citation indices). This proposed framework can serve as a template for assessing job candidates, for annual performance evaluation of current staff, and for career advancement (promotion and tenure). These practices can be adopted by institutions and individuals serving on hiring committees and providing peer-review of tenure and promotion dossiers. We note, however, that this shift in the valuation of activities for HRPT must be accompanied by training for supervisors in how to review performance equitably and account for disparities in the opportunities available to minoritized groups (i.e., invitations to collaborate and nominations for scholarly awards) as well as biases in the peer review process for publications and grants (Darling 2014; Grossman and DeVries 2019; Silbiger and Stubler 2019; Salerno et al. 2020), in citations (King et al. 2017), and in teaching evaluations (Lazos 2012; Mengel et al. 2019). This shift has already been proposed in the humanities, in the social sciences, and in medicine (Lieff 2009; Agate et al. 2020). In short, this is a framework for a new science-one that removes historic barriers and gatekeeping and recognizes all of the work needed for science to be diverse, equitable, and inclusive.

Our framework includes five broad, and equally important, categories of activities (discussed in greater detail below): Advancing scientific knowledge directly, Supporting advancement of (and access to) scientific knowledge, Using scientific knowledge for positive societal impact, Engaging society in science, and Serving science and society. The proportions of time divided among the categories are expected to vary between positions (e.g., for university professors, museum curators and collections staff, early career scientists, and those with more applied research programs) (Figure 1) and/orwithin rolesand between performance periods (i.e., faculty within a department may serve as PIs for research or training grants, faculty at primarily undergraduate institutions teach more courses, and faculty with a focus on DEI may be more directly involved with public outreach and work within professional societies) (Figure 2). Categories of activities and the proportion of effort invested (i.e., work time spent) can be visualized as a pie chart of a given individual's contributions and impact for any given time period. The allocation of effort can be agreed upon with a supervisor before the performance period or self-reported as part of the evaluation. Performance, or "scientific success", is then evaluated on the basis of that division of time/effort among categories, with deliverables for each category commensurate to the percent effort. Key performance metrics can be summed within categories for institutional annual reports but would appear alongside short narratives to provide context for the impact of activities and for the equity contributions that are difficult to directly measure. The result is a combination of qualitative and quantitative performance outcomes that highlight activities that advance scientific knowledge and improve the culture of science broadly, creating greater access, tackling social justice, and promoting DEI within our fields. These more detailed and precise accounts of effort and success can be shared as part of promotion or tenure decisions, providing a 

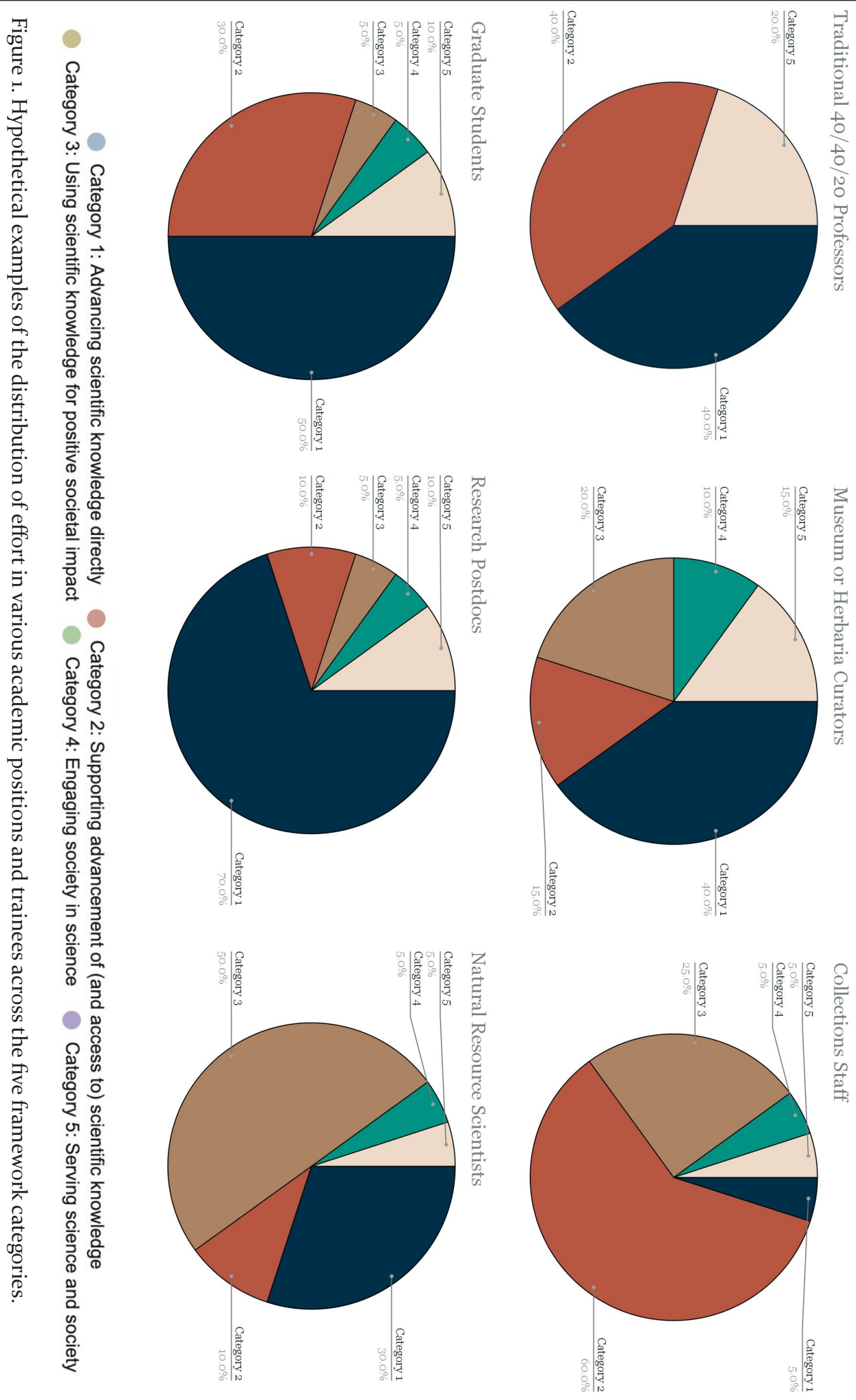


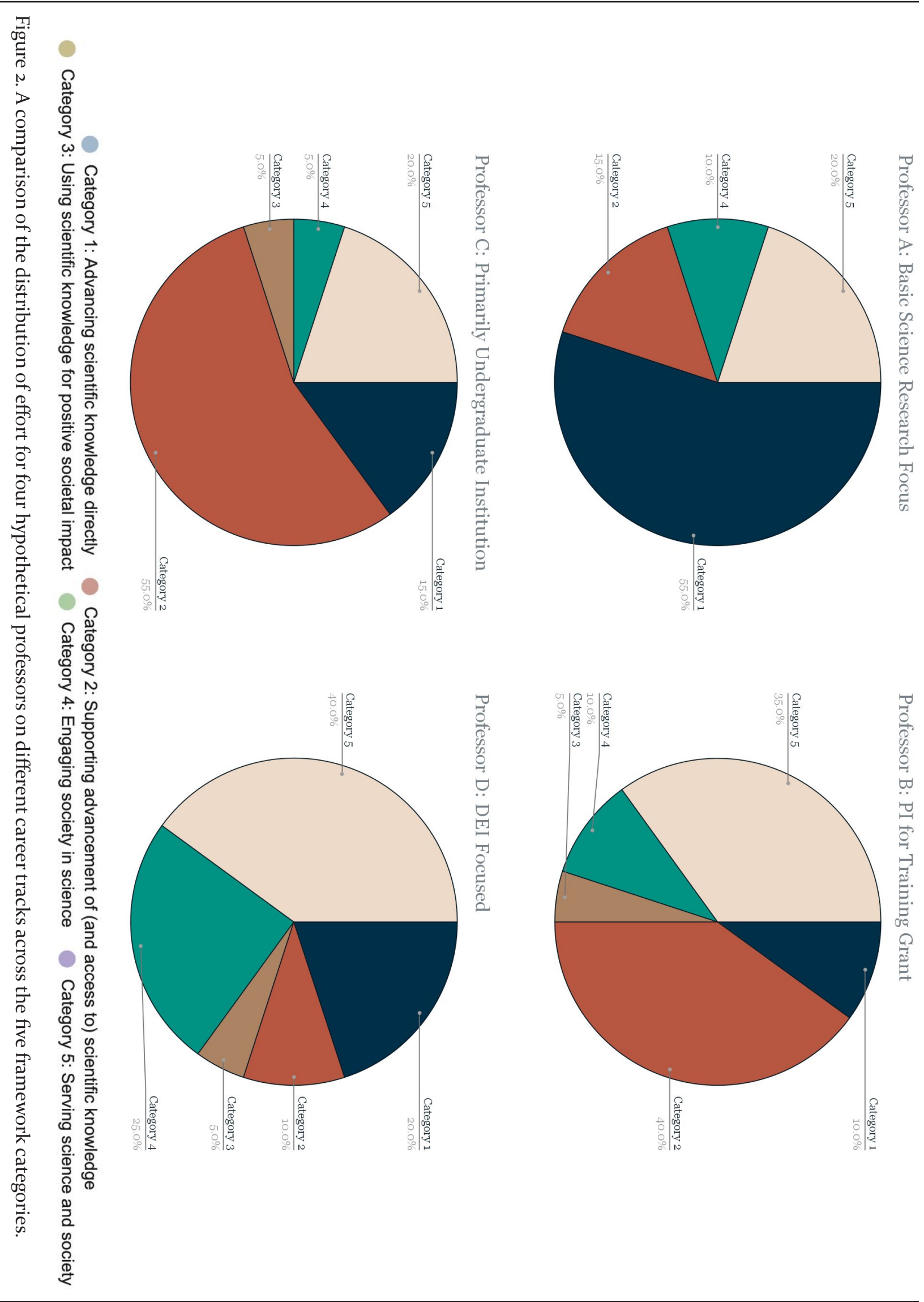


more accurate picture to external reviewers than the familiar " $40 / 40 / 20$ " percent split between broadly defined categories of research, teaching, and service for faculty.

\section{CATEGORY 1: ADVANCING SCIENTIFIC KNOWLEDGE DIRECTLY.}

This category is focused on activities associated with doing research individually and/or collaboratively: primary research, research dissemination, and fundraising/ grant-writing to support these activities.

This category encompasses the majority of the traditional scholarly metrics under which researchers are commonly evaluated. Deliverables could include peer-reviewed publications, research and publications about DEI, non-peer reviewed publications for scientific audiences (e.g., perspective/opinion editorials, book reviews, scholarly books or book chapters), presentations to scientific audiences, projects in earlier stages of completeness that are moving forward (i.e., not yet published, critical for recognizing the timescale of monographic work), and research grants. This could also include honors and awards received from academic institutions and professional societies. Quantitative metrics could include traditional academic metrics like number of publications, grants received, citation indices, etc. Qualitative outcomes could include publications co-authored with mentees, local researchers, or traditionalknowledge holders, or the advancement of work in a long-term project likea monograph.

3 CATEGORY 2: SUPPORTING ADVANCEMENT OF (AND ACCESS TO) SCIENTIFIC KNOWLEDGE.

This category is focused on many aspects of research that are difficult to directly quantify but critical for the advancement of science. This category can be further divided into four areas: community science, formal education, infrastructure development and support, and editorial and review service. Many of these activities are also traditionally considered in the formal review and advancement processes.

Examples of community science include hosting/coordinating volunteers, engaging communities through a community science project (e.g., iNaturalist project or a bioblitz). Examples of formal education include formal mentorship (e.g., early career researchers in your lab), university courses taught, curriculum development, teaching modules that include decolonization of taxonomy/monography in systematics or biodiversity courses, workshops organized/ led/taught, and grants submitted/awarded to support these activities. Examples of infrastructure development and support include creating databases, generating new natural history collections, providing access to collections (facilitating loans, hosting visitors), improving collections (digitization, data curation), providing identifications, hosting/developing/ curating platforms (e.g., AmphibiaWeb), developing computational pipelines (e.g., tracked on GitHub), and grants submitted/ awarded for infrastructure. Examples of editorial and review service include formal editorial/review/panel service (manuscripts, books, or grants edited/reviewed/refereed) and informal review/translation for nonmentee colleagues (manuscripts edited). This category could also include honors and awards received from academic institutions and professional societies. Due to the diverse areas covered by this category, both quantitative and qualitative descriptions of activities will be necessary to summarize the effort and level of expertise required as well 
as the breadth and depth of the impact (e.g., lecturing for an introductory biology course of 500 undergraduates versus teaching a hands-on advanced phylogenetics workshop for 30 early career researchers).

\section{CATEGORY 3: USING SCIENTIFIC KNOWLEDGE FOR POSITIVE SOCIETAL IMPACT.}

This category focuses on the direct societal impacts of science, including resource management reports/assessments, collaboration with communities, governments, and organizations to develop/ implement management recommendations.

Examples of using scientific knowledgeforpositivesocietalimpactinclude Federal, State, or Local Government policy work/management or reports/assessments, partnerships with organizations to develop/ implement management recommendations, service to or in partnership with NGOs, provision of expert testimony, and research that directly addresses social, cultural, environmental or climate justice. This area of contribution has been identified in the human and social sciences and deemed "knowledge transfer activities," which may involve consulting, patent development, or other forms of partnership (OlmosPeñuela et al. 2014). This category could also include honors and awards received from a governmental or policy-making institution. Quantitative assessments could include number of reports produced, species assessed for the IUCN RedList, or number of hours spent with legislators. However, qualitative assessments would provide a narrative for the overall impact of the activities and allow for a discussion of impact on marginalized communities.

\section{CATEGORY 4: ENGAGING SOCIETY IN SCIENCE.}

This category includes outreach activities that are both traditionally quantifiable, as well as those which are not, but all of the activities in this category involve direct (non-academic) public engagement.

Examples of engaging society in science include popular writing, speaking engagements, social media engagement, collections tours, formal pre-college education programs, informal education programs, and development or scientific review of exhibits. As with Category 2, both quantitative and qualitative descriptions of activities will be necessary to summarize the effort and level of expertise required as well as the breadth and depth of the impact (e.g., authoring a 2500 word article for popular science magazine versus a 30-minute guest appearance on the Ologies podcast). This category could also include honors and awards received from a public-facing organization.

\section{CATEGORY 5: SERVING SCIENCE AND SOCIETY.}

This category concerns all of the activities that keep the wheels of the scientific academy turning at an institutional level (often referred to as institutional service) and also those that improve the culture of science. Although there may be significant overlap between these two, an important distinction can be made between activities and responsibilities that primarily benefit a given institution with which a scientist is affiliated and those that develop and benefit science more broadly. Service within science in a broader capacity is likely to be overlooked in HRPT when compared to institutional service, which may be more regularly recognized and understood from 
the perspective of the institution in which an individual is employed.

Examples of serving science and society include institutional committee service (e.g., IACUC, graduate admissions), service to scientific organizations (e.g., advisory board member of a scientific nonprofit or institute), service to professional societies (e.g., elected and volunteer positions), fundraising/donor events, supervisor responsibilities, natural history collections maintenance, participation in professional development, leadership of or participation in initiatives/organizations that target visibility, awareness, or social justice (e.g., \#BlackinSTEM, 50o Women Scientists, 500 Queer Scientists), service on DEI or career panels, and speaking about topics in DEI. Several of these aspects can be easily quantified (e.g., number of committees or speaking engagements), but many of them require qualitative details to provide context for their impact to our institutions and the academy.

\section{CONCLUSION}

Our proposed framework is an important step towards shifting the valuation of activities for HRPT, but we acknowledge that several challenges for those doing work not reflected by traditional scientific metrics remain. Chief among these is bias in our review and social structures that typically reward Category 1 activities, parity in pay and opportunity based on differences in the share of proposed work in each category of the framework (e.g., teaching-intensive versus research-intensive faculty positions), a generally lowvaluation of DEI (both directly and by underestimating the time, labor, and trauma it may entail), and in differentiating levels of engagement or impact for some of the interpersonal or social interactions, particularly in Categories 2-5. Furthermore, these categories can only be successful in promoting a more inclusive framework for evaluating scientists if individual contributions are made clear, for instance by using CRediT Contributor Roles Taxonomy to specify individual contributions to scientific scholarly output (Allen et al. 2014). Similarly, individuals can contribute or participate in activities in Categories 2-5, but if this is done in a lackluster, noncommittal, or boxchecking manner, it could prove counter effective or, at best, effect no real change. This is apparent when "slacktivist" platitudes are contrasted with deliverable-oriented activism. Mentoring (most specifically indicated in Category 2) is another activity that can be done well or in a desultory way, and it remains difficult to differentiate the varied forms that this takes or account for harm that may result from culturally insensitive practices when evaluating effort and impact (e.g., Daniels et al. 2019; Gelles et al. 2019; Limeri et al. 2019; McGee 2020).

The persistence of racism and colonialism in the practices and culture of the natural sciences today necessitates visible and intentional work toward a more diverse, equitable, and inclusive scientific community. In the context of ecology, evolutionary biology, and biodiversity science, this is more necessary than ever if we aim to recruit the talent and diverse perspectives needed to address the current biodiversity crisis. The work to undo the harmful legacy in our disciplines is often the burden of those in historically excluded groups, who themselves experience those very same DEI challenges. In addition, this work has been largely unrecognized by existing evaluation metrics, which often rely on solely quantitative measures of academic impact. More broadly, there is little to no value placed on professional 
activities that support access to scientific knowledge, use this knowledge for positive societal impact, engage society in science, and provide service to science. We hope that our proposed framework addresses some of these shortcomings. In closing, we acknowledge our proposed framework and recommendations are framed in light of the challenges facing scientists based in the United States. Although we believe many of these challenges are universal, we encourage natural scientists from other regions to modify and adopt this framework for their local context.

\section{Acknowledgments}

We thank the California Academy of Sciences Institute for Biodiversity Science and Sustainability Anti-Racist Working Group for inspiring the creation of this framework and providing a context for it to be implemented. We would also like to thank participants in the Modernizing Monography workshops, who helped us to see the broader relevance of this work and provided an avenue for formal publication. The Modernizing Monography workshops were sponsored by an NSF grant (DEB-1839205) to M. Daly and F. Zapata.

\section{References}

Agate N, Kennison R, Konkiel S, Long CP, Rhody J, Sacchi S, Weber P. The transformative power of values-enacted scholarship. Humanit Soc Sci Commun. 2020;7(1):112. https://doi.org/10.1057/ S41599-020-00647-Z

Allen L, Brand A, Scott J, Altman M, Hlava M. Credit where credit is due. Nature. 2014;508(7496):312313. https://doi.org/10.1038/508312a

Alperin JP, Nieves CM, Schimanski LA, Fischman GE, Niles MT, McKiernan EC. Meta-Research: How significant are the public dimensions of faculty work in review, promotion and tenure documents? Elife. 2019;8:e42254. https://doi.org/10.7554/eLife.42254

Corneille M, Lee A, Allen S, Cannady J, Guess A. Barriers to the advancement of women of color faculty in STEM. Equality, Diversity and Inclusion: An International Journal. 2019;38(3):328348. https://doi. org/10.1108/EDI-09-2017-0199

Cronin MR, Alonzo SH, Adamczak SK, Nevé Baker D, Beltran RS, Borker AL, Favilla AB, Gatins R, Goetz LC, Hack N, and others. Anti-racist interventions to transform ecology, evolution and conservation biology departments. Nat Ecol Evol. 2021;5:1213-1223. https://doi.org/10.1038/s41559-021-01522-Z

Daniels HA, Grineski SE, Collins TW, Frederick AH. Navigating social relationships with mentors and peers: comfort and belonging among men and women in STEM summer research programs. CBE Life Sci Educ. 2019;18(2):ar17. https://doi.org/10.1187/ cbe.18-08-0150

Darling ES. Use of double-blind peer review to increase author diversity. Conser Biol. 2014;29(1):297299. https://doi.org/10.1111/cobi.12333

Das S, Lowe M. Nature read in black and white: Decolonial approaches to interpreting natural history collections. J Nat Sci Collect. 2018;6:414.

Davies S, Putnam H, Ainsworth T, Baum J, Bove C, Crosby S, Côté I, Duplouy A, Fulweiler R, Griffin A, and others. Shifting our value system beyond citations for a more equitable future. Preprints. 2021:2021020493.

Evangelista DE, Goodman A, Kohli MKK, Bondocgawa Mafla Mills STT, Samuel-Foo M, Sanchez Herrera M, Ware JL, Wilson M. Why Diversity Matters Among Those Who Study Diversity. Am Entomol. 2020;66(3):4249. https://doi.org/10.1093/ae/tmaao37

Fire M, Guestrin C. Over-optimization of academic publishing metrics: observing Goodhart's Law in action. Gigascience. 2019;8(6):p.gizo53. https://doi. org/10.1093/gigascience/gizo53

Gelles L, Villanueva I, Di Stefano M. "Mentoring is ethical, right?": Women graduate students \& faculty in science \& engineering speak out. Int J Gend Sci Technol. 2019;11(1):108133.

Gorneau JA, Ausich WI, Bertolino S, Bik H, Daly M, Demissew S, Donoso DA, Folk R, Freire-Fierro A, Ghazanfar S A, and others. Framing the Future for 
Monography: Improving Recognition, Support, and Access. Bull Soc Syst Biol. 2022;1(1):8328. https://doi. org/10.18061/bssb.vii1.8328

Graves Jr. JL. The race myth: why we pretend race exists in America. New York: Dutton; 2004.

Graves Jr. JL. Why the nonexistence of biological races does not mean the nonexistence of racism. Am Behav Sci. 2015;59(11):14741495. https://doi. org/10.1177/0002764215588810

Graves Jr. JL. African Americans in evolutionary science: where we have been, and what's next. Evol Educ Outreach. 2019;12(1):110. https://doi.org/10.1186/ s12052-019-0110-5

Grossman GD, DeVries DR. Authorship decisions in ecology, evolution, organismal biology and natural resource management: who, why, and how. Anim Biodivers Conserv. 2019;42(2):337346. https://doi. org/10.3280o/abc.2019.42.0337

Gruber T. Academic sell-out: how an obsession with metrics and rankings is damaging academia. J Mark High Educ. 2014;24(2):165177. https://doi.org/10.108o /08841241.2014.970248

Haraway D. (1984). Teddy bear patriarchy: Taxidermy in the garden of Eden, New York City, 1908-1936. Soc Text. 1984;(11):20-64. https://doi.org/10.2307/466593 Harley DA. Maids of academe: African American women faculty at predominately white institutions. J Afr Am Stud. 2008;12(1):1936. https://doi.org/10.1007/ s12111-007-9030-5

Jimenez MF, Laverty TM, Bombaci SP, Wilkins K, Bennett DE, Pejchar L. Underrepresented faculty play a disproportionate role in advancing diversity and inclusion. Nat Ecol Evol. 2019;3(7):1030-1033. https:// doi.org/10.1038/s41559-019-0911-5

King MM, Bergstrom CT, Correll SJ, Jacquet J, West JD. Men set their own cites high: Gender and self-citation across fields and over time. Socius. 2017;3:1-22. https://doi.org/10.1177/2378023117738903

Lazos SR. Are student teaching evaluations holding back women and minorities?: The perils of "doing" gender and race in the classroom. In: Eds Gutiérrez y Muhs G, Flores Niemann Y, González CG, Harris AP. Presumed Incompetent. Utah State University Press; 2012.
LieffSJ.Perspective:themissing linkinacademiccareer planning and development: pursuit of meaningful and aligned work. Acad Med. 2009;84(10):1383-1388. https://doi.org/10.1097/ACM.obo13e3181b6bd54 Limeri LB, Asif MZ, Bridges BH, Esparza D, Tuma TT, Sanders D, Morrison AJ, Rao P, Harsh JA, Maltese AV, and others. "Where's my mentor?!" Characterizing negative mentoring experiences in undergraduate life science research. CBE Life Sci Educ. 2019;18(4):p.ar61. https://doi.org/10.1187/cbe.19-02-0036

Maas B, Pakeman RJ, Godet L, Smith L, Devictor V, Primack R. Women and Global South strikingly underrepresented among top-publishing ecologists. Conserv Lett. 2021;14(4):e12797. https://doi. org/10.1111/conl.12797

McGee EO. Interrogating structural racism in STEM higher education. Educ Res. 2020;49(9):633-644. https://doi.org/10.3102/o013189X20972718

Mengel F, Sauermann J, Zölitz U. Gender bias in teaching evaluations. J Eur Econ Assoc. 2019;17(2):535566. https://doi.org/10.1093/jeea/jvxo57

Miner KN, January SC, Dray KK, Carter-Sowell AR. Is it always this cold? Chilly interpersonal climates as a barrier to the well-being of early-career women faculty in STEM. Equal Divers Incl Int J. 2019;38(3):225-245. https://doi.org/10.1108/EDI-07-2018-0127

Miriti MN. The elephant in the room: Race and STEM diversity. Bioscience. 2020;70(3):237-242. https://doi. org/10.1093/biosci/biz167

Olmos-Peñuela J, Castro-Martínez E, d'Este P. Knowledge transfer activities in social sciences and humanities: Explaining the interactions of research groups with non-academic agents. Res Policy. 2014;43(4):696-706. https://doi.org/10.1016/j. respol.2013.12.004

Olsen D, Maple SA, Stage FK. Women and minority faculty job satisfaction: Professional role interests, professional satisfactions, and institutional fit. J Higher Educ. 1995;66(3):267-293. https://doi.org/10. 1080/00221546.1995.11774780

Orfinger AB. Diversity Beyond Insects in Entomology and Allied Disciplines. J Insect Sci. 2020;20(5):4. https://doi.org/10.1093/jisesa/ieaao93

Salerno PE, Páez-Vacas M, Guayasamin JM, Stynoski 
JL. Correction: Male principal investigators (almost) don't publish with women in ecology and zoology. PLoS One. 2020;14(6):e0218598. https://doi. org/10.1371/journal.pone.0233803
Silbiger NJ, Stubler AD. Unprofessional peer reviews disproportionately harm underrepresented groups in STEM. PeerJ. 2019;7:e8247. https://doi.org/10.7717/ peerj. 8247
Submitted: 21 May 2021

Editor: Felipe Zapata

Managing Editor: Dinah Ward
The Bulletin of the Society of Systematic Biologists publishes peer reviewed research in systematics, taxonomy, and related disciplines for SSB members. The Bulletin is an Open Access Gold publication. All articles are published without article processing or page charges. The Bulletin is made possible by a partnership with the Publishing Services department at The Ohio State University Libraries. Information about SSB membership is available at https://www.systbio.org. Questions about the Bulletin can be sent to Founding Editor Bryan Carstens. 